\title{
The Malone Stoma With Antegrade Colonic Enemas for Chronic Constipation in Adults
}

\author{
Ganesh R. Deshmukha, ${ }^{\mathrm{a}}$, Raymond Laird ${ }^{\mathrm{b}}$
}

\begin{abstract}
The Malone stoma with antegrade colonic enemas (MACE) is a procedure predominantly described in the European literature for treatment of chronic constipation refractory to medical management. Chronic constipation may be secondary to colonic or rectal inertia, or a condition known as pelvic floor dysfunction. We describe a case of a 22-year-old female with chronic constipation secondary to severe rectal and colonic inertia. She had an unexpected congenital anatomic anomaly discovered intra-operatively which made a restorative proctocolectomy impossible. The MACE procedure was performed after an initial proctectomy with coloanal anastomosis, and the patient has now lived free of constipation for 6 years with an acceptable bowel regimen.
\end{abstract}

Keywords: Malone stoma; Chronic constipation; Antegrade colonic enema

\section{Introduction}

Chronic constipation refractory to medical management is a life altering condition. There are several surgical options to consider that have been well described in the United States, such as a total or segmental colectomy [1]. Morbidities and mortalities are associated with these invasive procedures. European literature describes a minimally invasive procedure that has been successfully used to treat children and adults with chronic constipation called the Malone stoma with antegrade colonic enemas [2,3]. Here we present a case where a laparoscopic Malone stoma with antegrade colonic

\footnotetext{
Manuscript accepted for publication September 6, 2013

${ }^{\mathrm{a}}$ Department of Colon and Rectal Surgery, Oakwood Medical Center, Dearborn, MI, USA

${ }^{\mathrm{b}}$ Department of Medical Education, Henry Ford Wyandotte Hospital, Wyandotte, MI, USA

${ }^{\mathrm{c} C o r r e s p o n d i n g ~ a u t h o r: ~ G a n e s h ~ R . ~ D e s h m u k h, ~} 3106$ South Wayne

Road, Wayne, MI 48184, USA. Email: grdoffice@ameritech.net

doi: http://dx.doi.org/10.4021/jmc1524e
}

enemas has resolved the patient's chronic constipation.

\section{Case Report}

L. K. is a 22-year-old African American female who presented with chronic constipation and recurrent fecal impaction for several years. Her past medical history, surgical history and social history were unremarkable. She was scheduled for a colonoscopy. Under sedation, fecal material in the rectum was palpable as a large abdominal mass extending to the level of the umbilicus. The patient was disimpacted and required hospitalization for further treatment. A gastrografin enema and computed tomography (CT) scan were normal except for a large dilated atonic rectum.

Further outpatient workup included a colonic transit study, defecating proctogram, and anal manometry. On the colonic transit study, all 24 markers were identified in the descending colon on day 5 , consistent with abnormal hindgut transit. The patient was unable to evacuate on the defecating proctogram. Anal manometry showed normal sphincter function, but reproducing a rectoanal inhibitory reflex was difficult due to the megarectum. The patient remained on a bowel regimen of $17 \mathrm{~g}$ of polyethylene glycol daily for two months. She had another episode of severe impaction requiring bimanual disimpaction under anesthesia. An anal myectomy was done. Ganglion cells were present throughout the specimen, which ruled out Hirschsprung's disease.

She was scheduled for an elective proctocolectomy with a J-pouch and diverting ileostomy. When the peritoneal cavity was opened, the anatomy was found to be abnormal. The bladder and uterus were intra-abdominal. The rectum was extremely dilated and full of hard stool. The sigmoid was very redundant. The small bowel was noted to be infantile and poorly developed with the mesentery too short to create a J-pouch. The procedure was changed to a total proctectomy with coloanal anastamosis and a diverting loop colostomy in the proximal redundant sigmoid colon. Her post-operative course was uneventful and the colostomy functioned well. However, a colonic transit study that was performed with the loop colostomy in place was abnormal. After explaining the inherent risks, she underwent a colostomy reversal two 
months later. Her chronic constipation returned as expected, and another colonic transit study was performed. Twentyone rings were retained in the transverse colon on day 5 , again consistent with colonic inertia. A gastrografin enema was done because the patient had not had a bowel movement in 2 weeks. There was no mechanical obstruction, but there was an anastomotic stricture. She had a sigmoidoscopy with dilation of the coloanal stricture. Her bowel regimen with daily polyethylene glycol was continued and she was symptom free for 6 months. She developed fecal incontinence, so her bowel regimen was modified. Her severe constipation returned and continued for the next 3 months. A laparoscopic Malone stoma was performed for antegrade colonic enemas.

Six years have passed since the laparoscopic Malone ostomy was created. The appendiceal ostomy has been used with a bowel regimen of one liter of warm water irrigation on days 1 and 2 followed by polyethylene glycol on the 3rd day, then repeated on a continual basis. The patient has had successful treatment of her colonic inertia with this regimen with no residual constipation or fecal incontinence.

\section{Review of literature}

The Malone stoma was first described for children in 1990 in England. The 21 children studied had either chronic constipation or fecal incontinence. Mean age of the children in the study was 12 years old, ranging from 18 months to 18 years. Fifteen of 21 children showed significant improvement, with 12 of these patients showing complete resolution of constipation or fecal incontinence [4]. Other studies have shown success rates of treatment with the Malone stoma with antegrade enemas to vary from 50-95\% [5-9]. This technique has been shown to be a safe and acceptable treatment option for chronic constipation and fecal incontinence. Many children also achieve high functional scores with this technique. According to Schell et al, $85 \%$ of patients achieved the maximum benefit from the surgery with a 6-year follow-up period [10]. Although the original surgery was conducted in children, adults have had successful treatment with this procedure in repeated studies $[3,5,11]$.

The stoma may be created by either laparoscopic or open surgical procedures. Two 5 -mm ports may be used to eviscerate the appendix in the right lower quadrant. One port is for the camera, and the other port is for a bowel grasper. A third port may be placed if needed. A small Rocky Davis incision may be used in an open technique to eviscerate the appendix. The appendix is brought out through a skin incision and an ostomy is fashioned by securing the mucosal surface to the skin with absorbable 3-0 sutures. If an appendectomy has been previously done, a cecal flap or sleeve may be created with a stapling device and used for the stoma. There are several different techniques for creating the stoma $[5,12,13]$. An $18 \mathrm{Fr}$ catheter is used to intubate the lumen of the appendix and is left in place for $2-4$ weeks until a well healed tract is established $[14,15]$. The stoma requires daily intubation in order for the tract to remain patent. The patient intubates the stoma and allows the bowel irrigant to lavage the colon. After the lavage is complete, the catheter is removed and either a band aid or small gauze is used to cover the stoma. Stomal stenosis is the most common complication associated with this procedure and is most often treated by dilating the tract with Hegar dilators [11]. Other complications include stomal leakage (3\%), channel obliteration (3\%), stomal prolapse (1\%), infection, pain and bleeding $[6,16]$.

The bowel regimen is individually tailored. Some patients require 500 to $2000 \mathrm{~mL}$ of water to be flushed through the stoma daily $[3,11]$. This is usually alternated with polyethylene glycol ranging from every other day to once every several weeks [6]. The bowel regimen is altered until a successful treatment is established [5]. The average time from instilling the solution until complete colonic emptying is 30 to 60 minutes $[3,5,10,13]$.

\section{Discussion}

The Malone stoma is a rarely utilized surgical procedure in the United States for chronic constipation, however this technique should be in every surgeon's armamentarium. This procedure is safe and effective for the treatment of chronic constipation [10]. Patients with significant health comorbidities tolerate this minimally invasive procedure well. This makes the procedure more attractive for elderly patients who may not be able to undergo a major abdominal operation, such as a total colectomy. This is also an option for patients with pelvic floor dysfunction, and combined colonic and rectal inertia. The author has performed several restorative proctocolectomies for inertia; however the patients often have significant morbidity from continued pelvic floor dysfunction [17]. These patients show little to no improvement in symptoms from a traditional proctocolectomy.

This procedure is well described in Europe and several studies have been conducted to evaluate the long term effectiveness of the surgery. Most of the literature describes the surgery on children; however the Malone stoma has been successfully used to treat constipation in adults $[3,5,11]$. The procedure can have a fair number of complications, and as always, the risks need to be weighed against the benefits for every individual $[6,16]$. More studies need to be conducted in the United States to determine the patient population that would have the most benefits from the surgery.

In conclusion, the Malone stoma with antegrade continence enemas is an effective treatment for adult patients with chronic constipation and fecal incontinence. This 22-year-old patient's medical options for her chronic constipation had been exhausted. Surgery was her only chance for a normal lifestyle. Her infantile anatomy made the initial proposed surgery impossible to perform. This patient was an 
ideal candidate for the Malone stoma with antegrade colonic enemas. She has now been free of constipation for 6 years and enjoys a normal lifestyle with her daily bowel regimen. This technique has been utilized for pelvic floor dysfunction, as well as colonic and rectal inertia with good results. The bowel regimen is tailored for each patient. More studies need to be performed in the United States, but this European surgery should be considered for the treatment of intractable chronic constipation.

\section{Disclaimers}

Dr. Ganesh Deshmukh and Raymond Laird report neither conflicts of interest nor financial ties.

\section{Authorship Credit}

Ganesh Deshmukh, MD: concept and design, acquisition and interpretation of data, revision of article critically and final approval of paper to be published.

Raymond Laird, DO: concept and design, acquisition and interpretation of data, drafting of the article and final approval of paper to be published.

\section{References}

1. Pemberton JH, Rath DM, Ilstrup DM. Evaluation and surgical treatment of severe chronic constipation. Ann Surg. 1991;214(4):403-411; discussion 411-403.

2. Griffiths DM, Malone PS. The Malone antegrade continence enema. J Pediatr Surg. 1995;30(1):68-71.

3. Lefevre JH, Parc Y, Giraudo G, Bell S, Parc R, Tiret E. Outcome of antegrade continence enema procedures for faecal incontinence in adults. Br J Surg. 2006;93(10):1265-1269.

4. Malone PS, Ransley PG, Kiely EM. Preliminary report: the antegrade continence enema. Lancet. 1990;336(8725):1217-1218.

5. Gerharz EW, Vik V, Webb G, Leaver R, Shah PJ, Woodhouse CR. The value of the MACE (Malone antegrade colonic enema) procedure in adult patients. J Am Coll Surg. 1997;185(6):544-547.

6. King SK, Sutcliffe JR, Southwell BR, Chait PG, Hutson JM. The antegrade continence enema successfully treats idiopathic slow-transit constipation. J Pediatr Surg. 2005;40(12):1935-1940.

7. Curry JI, Osborne A, Malone PS. The MACE procedure: experience in the United Kingdom. J Pediatr Surg. 1999;34(2):338-340.

8. Curry JI, Osborne A, Malone PS. How to achieve a successful Malone antegrade continence enema. J Pediatr Surg. 1998;33(1):138-141.

9. Levitt MA, Soffer SZ, Pena A. Continent appendicostomy in the bowel management of fecally incontinent children. J Pediatr Surg. 1997;32(11):1630-1633.

10. Schell SR, Toogood GJ, Dudley NE. Control of fecal incontinence: continued success with the Malone procedure. Surgery. 1997;122(3):626-631.

11. Krogh K, Laurberg S. Malone antegrade continence enema for faecal incontinence and constipation in adults. Br J Surg. 1998;85(7):974-977.

12. Thomas K, Bassuini M. Laparoscopic caecodivision ACE (antegrade continence enema) procedure. Tech Coloproctol. 2008;12(1):65-67.

13. Malone PS, Curry JI, Osborne A. The antegrade continence enema procedure why, when and how? World $\mathrm{J}$ Urol. 1998;16(4):274-278.

14. Karpman E, Das S, Kurzrock EA. Laparoscopic antegrade continence enema (Malone) procedure: description and illustration of technique. J Endourol. 2002;16(6):325-328; discussion 328.

15. Robertson RW, Lynch AC, Beasley SW, Morreau PN. Early experience with the laparoscopic ace procedure. Aust N Z J Surg. 1999;69(4):308-310.

16. Bani-Hani AH, Cain MP, Kaefer M, Meldrum KK, King S, Johnson CS, Rink RC. The Malone antegrade continence enema: single institutional review. J Urol. 2008;180(3):1106-1110.

17. Bernini A, Madoff RD, Lowry AC, Spencer MP, Gemlo BT, Jensen LL, Wong WD. Should patients with combined colonic inertia and nonrelaxing pelvic floor undergo subtotal colectomy? Dis Colon Rectum. 1998;41(11):1363-1366. 